\title{
Síndrome dos edifícios doentes em bancários
}

\author{
Sick building syndrome in bank-clerks
}

\author{
Ubiratan P. Santos*, Davi Rumel**, Norton A. Martarello***, Clara S. W. Ferreira**, Marcos Paiva \\ Matos****
}

\begin{abstract}
SANTOS, U. P. et al. Síndrome dos edifícios doentes em bancários. Rev. Saúde públ., S.Paulo, 26: $400-4,1992$. Foram identificadas associações entre conforto ambiental e sinais e sintomas entre trabalhadores expostos a ambientes fechados, ou seja, ambientes com ventilação e climatização artificiais. A população de estudo compreendeu 312 trabalhadores bancários distribuídos em dois edifícios localizados na Cidade de São Paulo. O delineamento é transversal com questionários autodirigidos, e a técnica de análise foi a regressão logística. A Síndrome dos Edifícios Doentes definida pela Organização Mundial da Saúde foi dividida em uma síndrome de sintomas gerais e em uma síndrome de sintomas de irritação de membrana mucosa. Foram relevantes para sintomas gerais, o sexo feminino, a organização do trabalho e a temperatura inadequada. Para sintomas de irritação de membrana mucosa, foram relevantes o sexo feminino e a temperatura inadequada. A organização do trabalho foi relevante para absenteísmo.
\end{abstract}

Descritores: Exposição ambiental. Doenças ocupacionais, epidemiologia. Poluição do ar, efeitos adversos.

\section{introduçáo}

Em 1982, o Comitê Técnico da Organização Mundial da Saúde definiu a síndrome do edifício doente (SED) como o conjunto dos seguintes sintomas: dor de cabeça; fadiga; letargia; prurido e ardor nos olhos, irritação de nariz e garganta; anormalidades na pele e falta de concentração, em trabalhadores de escritórios ${ }^{6,10}$.

Esta síndrome está relacionada a avanços tecnológicos nos sistemas mecânicos de ventilação e climatização, aliado ao interesse econômico de plena utilização do terreno nas regiões centrais das grandes cidades (esses edifícios permitem a eliminação de vãos centrais utilizados para iluminação e ventilação).

A arquitetura avança com estas novas possibilidades projetando altos edifícios com muito vidro, todos fechados, que vão fazer parte dos cartões postais de algumas cidades. As paredes internas

\footnotetext{
* Secretaria de Higiene e Saúde de Santos - Santos, SP.

** Departamento de Epidemiologia da Faculdade de Saúde Pública da USP - São Paulo, SP

*** Programa de Saúde do Trabalhador da Zona Norte da Secretaria de Saúde do Estado de São Paulo - São Paulo, SP.

**** Fundação Jorge Duprat Figueiredo de Segurança e Medicina do Trabalho - São Paulo, SP.
}

Separatas/Reprints: D. Rumel - Av. Dr. Amaldo, 715 - 01246 904 - São Paulo, SP - Brasil.

Publicação financiada pela FAPESP. Processo Saúde Coletiva 91/4994-0. desaparecem permitindo maior flexibilidade na organização e no controle do trabalho. Unidades de climatização e de ventilação operados manualmente desaparecem do cenário dos escritórios diminuindo a sobrecarga elétrica na fiação e consequientemente os riscos de incêndio.

A possibilidade de criar microclimas artificiais permite obter condiçð̃es de conforto em situaçð̃es de temperaturas extremas. Edifícios fechados proliferam no Alasca e na Arábia Saudita. Num país de clima tropical esses ambientes podem proteger os trabalhadores dos efeitos adversos do calor.

Fornecer um ambiente apropriado e agradável dentro de edifícios aos seus usuários, foi sempre um objetivo implícito em projetos arquitetônicos. Entretanto, os edifícios totalmente fechados incapacitaram os ocupantes a fazerem alteraçōes voluntárias no ambiente interno para adequá-lo às suas necessidades.

Em clima tropical como o da cidade de São Paulo, o vidro facilita a penetração das ondas de calor no interior dos edifícios. O posicionamento do sol em relação às faces do edifício pode provocar uma maior irradiação de calor em um dos lados em detrimento do outro.

A poluição das ruas causadas pelo tráfego dos automóveis podem contaminar o ar respirado pelos ocupantes de escritórios localizados no subsolo ou primeiros andares de edifícios quando o funil de entrada de ar para esses ambientes localiza-se ao nível da rua.

Durante o mesmo periodo proliferaram novos materiais sintéticos para a construção civii : mobi- 
liário e novos equipamentos de escritório, introduzindo significante, porém não reconhecida, fonte de contaminação interna. Acrescenta-se a esta contaminação a proveniente dos materiais de limpeza, fotocopiadoras, fumaça de cigarros e efluentes do corpo.

Esses edifícios tornaram-se dependentes de um amplo consumo de energia para o seu sistema de ventilação mecânica e iluminação artificial, criando uma demanda por novas fontes e transferindo aos consumidores o excesso de gasto com energia. Tentativas de minimizar os custos levam a um aumento na proporção de ar reciclado e diminuição da troca de filtros e limpeza de ductos de ventilação, levando a aumento da poluição do ambiente interno e 0 aparecimento de microorganismos que passam a se desenvolver em quantidade no sistema de refrigeração e umidificação.

O ambiente do edifício é multi-facetado. Inclui componentes como temperatura, iluminação, nível de ruído, características físicas do edifício, assim como o uso do espaço. A qualidade do ambiente envolve todos esses fatores. Devido à interação dos mesmos e ao fato de que o significado dos vários aspectos varia de pessoa a pessoa e de atividade a atividade, é difícil definir qualidade ambiental em termos precisos.

A qualidade do meio afeta direta e indiretamente o bem-estar, o temperamento e o rendimento das pessoas quando desenvolvem suas atividades. A impressão geral que os ocupantes têm de seu próprio ambiente fará com que se sintam confortáveis e aceitem o espaço, o que trará repercussões positivas à saúde e produtividade.

Se os ocupantes de um edifício de escritórios estão satisfeitos com seu meio interno, diminuem as queixas relacionadas à saúde, o número de ausências injustificadas e o trabalho se torna mais eficaz. Isto foi demonstrado em um estudo levado a cabo com trabalhadores de escritório antes e depois da empresa se mudar para um novo edifício com más condições ambientais. Houve um grande aumento do absenteísmo devido a queixas relacionadas à saúde e conforto após a mudança ${ }^{8}$.

Para a monitoragem ambiental e avaliação de edifícios de microclima artificial, alguns pesquisadores propõem as seguintes mensuraçōes ${ }^{1}$ : taxa de renovação do ar e concentração do $\mathrm{CO}$ como indicador de ventilação adequada, concentração de CO como indicadores de poluição externa, temperatura e umidade como indicadores de conforto térmico, concentração de partículas respiráveis em suspensão como indicador de eficiência do filtro e presença de fumaça de cigarro, número de colônias de bactérias (ágar soja-tripticase) e fungos (ágar de rosa de Bengala) como indicadores de contaminação microbiológica.

Fanger $^{2}$ tem uma abordagem mais qualitativa a respeito desta questão. Em sua opinião o ser humano é o último juiz da qualidade do ar. "No ar interno, milhares de compostos químicos estão presentes. Só a fumaça do cigarro é composta de aproximadamente de 50.000 substâncias químicas. Bioefluentes dos seres humanos e gases ou vapores de materiais de construção ou escritório são da mesma ordem de magnitude. Estes milhares de compostos podem estar presentes numa concentração tão pequena que é difícil ser detectado pelas presentes técnicas de análise química. Algumas destas substâncias estão acima do limite de odor e contribuem para a sensação de ar estafante. Há mais de meio milhão de compostos orgânicos com odor e muitos devem ter um baixo limite de odor. Atualmente temos conhecimento sobre a irritação e o odor de alguns compostos, alguma informação sobre a mistura de 2 compostos, mas nenhuma informação que nos faça predizer sobre a mistura de milhares de compostos orgânicos, mesmo que seja possível analisar a exata composição da mistura. Como identificar e quantificar a qualidade do ar em ambientes fechados quando a química não tem condiçōes de nos ajudar? A resposta está no uso do homem como medida. $O$ nariz do homem é o instrumento mais sensível de avaliação".

A partir deste raciocínio, Fanger ${ }^{2}$ criou uma unidade de medida de poluição do ar denominada "olf" (do latim olfactus). $O$ "olf" é semelhante ao lumen, unidade de energia de luz, e ao watt, unidade de energia do ruido.

A mensuração da quantidade de "olfs" depende da fonte padrão e da velocidade de fluxo do ar. A fonte padrão é uma pessoa sedentária com 1 met (taxa de metabolismo basal) exposta ao fluxo de ar de $10 \mathrm{I} / \mathrm{s}$, cujo odor é detectado por uma amostra de cheiradores devidamente treinados. Os cheiradores equivalem aos provadores de vinho ou café. Utiliza-se este instrumento de mensuração por não existir instrumento técnico que os substitua por enquanto. Esta medida de percepção de odor denomina-se "decipol" que é igual a 0,1 olf $(\mathrm{I} / \mathrm{s})$. $\mathrm{O}$ "decipol" é semelhante ao lux (unidade de mensuração de iluminação), e ao decibel (unidade de mensuração do ruído).

Esta metodologia foi aplicada na avaliação de 20 edifícios com microclimatizaçāo artificial, onde as medidas de concentração de $\mathrm{CO}_{2}, \mathrm{CO}$, material particulado respirável, total de substâncias orgânicas e fluxo de ar estavam dentro de padrōes de normalidade 3 .

Os autores identificaram a medida de oito "olfs" por ocupante distribuidos da seguinte forma: 3 a 4 provenientes do material existente no espaço, 3 a 4 provenientes do sistema de ventilação e 1 proveniente da fumaça de cigarro. $O$ fluxo de ar era em média $27 \mathrm{I} / \mathrm{s}$ por ocupante, acima dos padrões de 
normalidade. Mas se considerarmos os 8 "olfs" por ocupante, o fluxo seria de $3 \mathrm{I} / \mathrm{s}$ para cada ocupante, abaixo dos padrões de normalidade e poderiam justificar muitas queixas da SED.

No Brasil, este assunto começa a ser cada dia mais discutido pela imprensa e revistas científicas ${ }^{9}$. Opresente trabalho procura mostrar que este problema jáafeta trabalhađores brasileiros.

\section{Materlal e Método}

A população de estudo compreendeu 312 trabalhadores bancários, 179 homens e $133 \mathrm{mu}$ lheres, 217 de uma empresa e 95 de outra, com local de trabalho em dois prédios distintos, ambos fechados. Um edifício está localizado na região central e outro na região da zona norte da cidade de São Paulo, SP. Em ambos a ventilação interna é mantida artificialmente por equipamento central de troca e refrigeração de ar tipo FANCOIL, iluminação artificial durante o dia e pisos acarpetados. Optou-se por uma análise conjunta de dados das duas empresas com o intuito de obter significância dos dados.

Com a devida autorização das empresas e concordância dos trabalhadores, foi distribuído questionário* para ser respondido pelos trabalhadores sem necessidade de identificação, tomando por base um outro semelhante usado por empresa canadense especializada em avaliaçðes ambientais ${ }^{5}$.

A coordenação e execução do trabalho de campo esteve a cargo da equipe do Programa de Saúde do Trabalhador da Zona Norte da Cidade de São Paulo - Secretaria do Estado da Saúde.

Foi considerado doente com síndrome de sintomas gerais quem assinalou a alternativa "sempre" à presença de qualquer um dos seguintes sinais e sintomas nos últimos 6 meses: dor de cabeça, tontura, fadiga, sonolência, fraqueza e naúsea. Foi considerado doente com síndrome de sintomas de irritação da membrana mucosa quem assinalou a alternativa "sempre" à presença de qualquer um dos seguintes sinais e sintomas nos últimos 6 meses: falta de ar ou chiado no peito, ardor ou irritação nos olhos, dor ou irritação na garganta, irritação nasal, gripe ou resfriado e pele seca ou descamativa. Foi considerado faltoso a quem não compareceu a um dia de trabalho por motivos de saúde nos últimos 6 meses.

As variáveis de exposição foram: ar muito abafado, umidade do ar adequada e temperatura adequada. Foram considerados expostos quem assinalou a alternativa "sempre" para ar muito abafado, e

\footnotetext{
* A disposição dos interessados, com os autores do trabalho.
}

quem assinalou a alternativa "nunca" para umidade de ar e temperatura adequados. Medidas ambientais não foram analisadas por compartilharmos da opinião de Fanger ${ }^{2}$, já exposta na introdução.

Possíveis variáveis de confusão ("confounding") foram analisadas: sexo, tempo de serviço, idade e satisfação com a organização do trabalho ${ }^{7}$. Foi considerada organização do trabalho inadequada a quem assinalou a alternativa "nunca" para a questão: "o seu trabalho lhe dá satisfação?", em uma sequiência de perguntas sobre o processo de organização do trabalho.

Pelo fato de previamente à coleta de dados os pesquisadores não conhecerem nem a distribuição dos fatores de exposição, nem a distribuição das variáveis de efeito na população de estudo, este delineamento de pesquisa é caracterizado como transversal.

A análise dos dados foi realizada com os programas EPI INFO e EGRET.

\section{Resultados e Discussáo}

A Tabela 1 apresenta as medidas de significância (probabilidade de erro alfa) dos possíveis fatores de risco analisados de forma isolada em relação a sintomas gerais, sintomas de irritação de membrana mucosa e absenteísmo.

Tabela 1. Medidas de significância $(p \alpha)$ resultantes de testes de associação entre prováveis fatores de risco à sindrome dos edifíficios doentes (sintomas gerais e irritação de mucosa) e absenteismo.

\begin{tabular}{|c|c|c|c|}
\hline $\begin{array}{l}\text { Efeito S } \\
\text { Variáveis } \\
\text { de Exposição }\end{array}$ & $\begin{array}{l}\text { Sintomas } \\
\text { Gerais }\end{array}$ & $\begin{array}{l}\text { Irritação de } \\
\text { Membrana } \\
\text { Mucosa }\end{array}$ & Absenteísmo \\
\hline $\begin{array}{l}\text { Idade* } \\
\text { Tempo de Serviço* } \\
\text { Sexo Feminino** } \\
\text { Organização do }\end{array}$ & $\begin{array}{l}0,11 \\
0,80 \\
0,05\end{array}$ & $\begin{array}{l}0,17 \\
0,30 \\
0,04\end{array}$ & $\begin{array}{l}0,29 \\
0,67 \\
0,23\end{array}$ \\
\hline $\begin{array}{l}\text { Trabalho** } \\
\text { Ar abafado** } \\
\text { Temperatura }\end{array}$ & $\begin{array}{l}0,00 \\
0,00\end{array}$ & $\begin{array}{l}0,36 \\
0,02\end{array}$ & $\begin{array}{l}0,07 \\
0,09\end{array}$ \\
\hline $\begin{array}{l}\text { Inadequada"* } \\
\text { Umidade Excessiva*" }\end{array}$ & $\begin{array}{r}0,00 \\
0,01\end{array}$ & $\begin{array}{l}0,01 \\
0,30\end{array}$ & $\begin{array}{l}0,08 \\
0.18\end{array}$ \\
\hline
\end{tabular}

- Kruskral-Wallis para 2 grupos

** Qui-quadrado

Ser do sexo feminino apresentou associação significante com sintomas gerais e sintomas de irritação da membrana mucosa. Estes resultados são compatíveis com dados de literatura ${ }^{4}$. Parece que as mulheres são mais sensíveis a estas queixas ou expressam mais que os homens os sintomas. 
Tabela 2. Medidas de significância $(p \quad \alpha)$ e de intensidade de associação (OR) das análises de regressāo logística para prováveis fatores de risco à sindrome dos edifícios doentes (sintomas gerais $\theta$ irritação de membrana mucosa) e absenteísmo.

\begin{tabular}{|c|c|c|c|c|c|c|}
\hline \multirow{2}{*}{$\begin{array}{l}\text { Variáveis } \\
\text { de Exposição }\end{array}$} & \multicolumn{2}{|c|}{ Sintomas Gerais } & \multicolumn{2}{|c|}{$\begin{array}{c}\text { Irritação de } \\
\text { Membrana Mucosa }\end{array}$} & \multicolumn{2}{|c|}{ Absenteísmo } \\
\hline & $p \alpha$ & OR & $p \alpha$ & OR & $p \alpha$ & OR \\
\hline $\begin{array}{l}\text { Sexo Feminino" } \\
\text { Organização do Trabalho* } \\
\text { Ar abafado* } \\
\text { Temperatura Inadequada* } \\
\text { Umidade Excessiva* }\end{array}$ & $\begin{array}{l}0,00 \\
0,00 \\
0,30 \\
0,07 \\
0,76\end{array}$ & $\begin{array}{l}3,48 \\
8,34 \\
1,68 \\
2,83 \\
1,18\end{array}$ & $\begin{array}{l}0,03 \\
0,29 \\
0,11 \\
, 1,1\end{array}$ & $\begin{array}{l}1,84 \\
1,46 \\
1,85 \\
, \ldots\end{array}$ & $\begin{array}{l}0,03 \\
0,26 \\
0,08 \\
\ldots,\end{array}$ & $\begin{array}{l}1,1,92 \\
0,69 \\
0,53 \\
,,,\end{array}$ \\
\hline & & & , , & ,', & ' & $\cdots$ \\
\hline
\end{tabular}

- Razão de verossimilhança

Idade e tempo de serviço não apresentaram associaçōes significantes. Satisfação com a organização do trabalho teve associação significante com sintomas gerais e absenteísmo. A percepção de ar abafado e temperatura inadequada tiveram associaçōes significantes com sintomas gerais, sintomas de irritação de membrana mucosa e absenteísmo. A percepção de umidade inadequada, teve associação significante com sintomas gerais.

A Tabela 2 apresenta as medidas de "odds ratio" (estimativas de risco relativo) e de significância dos resultados da análise de regressão logística. Foram incluídos nos modelos os possiveis fatores de risco que foram significantes $(p<0,10)$ nas análises com fatores isolados conforme resultados já expressos acima.

Para sintomas gerais, permaneceram no modelo final as variáveis sexo feminino, satisfação com a organização do trabalho e temperatura inadequada, para sintomas de irritação de membrana mucosa, permaneceram sexo feminino e temperatura inadequada para absenteísmo permaneceu satisfação com a organização do trabalho (temperatura inadequada teve um $\mathbf{p}$ alfa de 0,08 , porém como fator de proteção, o que levou a desconsiderar este resultado).

Cabe assinalar que na empresa com 95 trabalhadores, $18 \%$ apresentam sintomas gerais permanentemente, $33 \%$ apresentam sintomas de irritação de membrana mucosa permanentemente e $39 \%$ faltaram ao menos um dia de trabalho no espaço de 6 meses por motivos de saúde. $\mathrm{Na}$ empresa com 217 trabalhadores, $9 \%$ apresentam sintomas gerais permanentemente, $23 \%$ apresentam sintomas de irritação de membrana mucosa permanentemente e $52 \%$ faltaram ao menos um dia de trabalho no espaço de 6 meses por motivos de saúde. As percentagens elevadas indicam a relevância do problema em estudo.
A percepção ambiental de que a temperatura é inadequada e a insatisfação com a organização do trabalho são fatores determinantes à má qualidade de vida dos trabalhadores nas empresas.

O questionário utilizado mostrou ser um instrumento útil para detectar desequilibrios no bemestar de indivíduos expostos a condições ambientais e de trabalho desfavoráveis. Os edifícios fechados, com ventilação e climatização artificial nem sempre conseguem criar um ambiente de conforto a seus usuários, apesar de terem sido construídos para tal finalidade.

\section{Conslderaçóes Finais}

A tentativa de se proteger da poluição externa, muitas vezes é derrotada pela poluição interna. $O$ controle da poluição externa pode ser resolvido de forma coletiva, pois afeta toda a cidade e não só os trabalhadores de um edifício.

A garantia de um conforto térmico ambiental é questionável pelo aumento de consumo de energia para manter um prédio constantemente ventilado, iluminado, e numa mesma temperatura. Além do mais a capacidade de consumo energética não é infinita e cada vez se toma mais cara e mais agressiva ao ambiente a obtenção de novas fontes de energia.

A impossibilidade de controle da temperatura e ventilação pelo próprio indivíduo é uma agressão a sua individualidade. Dependendo do dia, tipo de trabalho, vestuário e características pessoais, as condições ambientais ideais variam.

A centralização de todos os departamentos num único prédio cada vez menos é justificada pela modema tecnologia das comunicações.

Em situaçōes de temperatura extrema, se justifica o acionamento de pequenas unidades de climatização ou um sistema de climatização central. 
Mas isto seria transitório e permitiria renovação do ar periodicamente.

A insatisfação com a organização do trabalho não foi resolvida nos edifícios fechados. Pode ser que trabalhar em grandes salas sob supervisão permanente agravem essa insatisfação.

No Brasil já encontramos muitos edifícios fechados nos principais corredores comerciais das grandes cidades, e até em cidades menores que têm enormes espaços para grandes construçð̌es.

Há riscos à saúde dos ocupantes desses edifícios, afetando, provavelmente, à atividade desses trabalhadores, como o presente estudo demonstrou.

Considerando os comentários acima, julgamos que arquitetos, incorporadores, empresários, sindicatos e poder público deveriam avaliar todas as implicaçð̃es da construção de edifícios fechados ao nosso cenário urbano.

Os serviços de higiene e segurança do trabalho de empresas localizadas em edifícios fechados deveriam implantar um sistema de vigilância epidemiológica específicos à sindrome dos edifícios doentes para orientar a intervenção em áreas de exposição específicas.

A aplicação da metodologia do presente trabatho em diferentes edifícios pode ser instrumento útil de organização dos trabalhadores do setor de serviços para lutar por melhores condições de trabalho.

SANTOS, U.P. et al. [Sick building syndrome in bankclerks]. Rev. Saúde públ., S.Paulo, 26: 400-4,1992. This study seeks, for the first time, to identify the sick building syndrome in Brazil. The study population consisted of 312 bank-clerks distributed in two closed buildings situated in S.Paulo city, Brazil. Data were collected by questionnaire, the design was cross-sectional and analysis made by logistic regression. Female, job satisfaction and inappropriate temperature were related to general symptoms. Female and inappropriate temperature were
Ielated to mucus membrane symptoms. Job satisfaction was related to absenteeism.

Keywords: Environmental exposure. Occupational diseases, epidemiology. Air poluttion, adverse effects.

\section{Referências Bibliográficas}

1. COLLET, C. \& STERLNNG, E. Estratégia de investigação para o diagnóstico e resolução dos problemas causados pela qualidade de ar em escritórios. Rev. bras. Saúde ocup., $18(70): 59-61,1990$.

2. FANGER, P.O. The olf and decipol. ASHRAE J., oct 1988:35-8. [Cópia xerox].

3. FANGER, P.O. Hidden olfs in sick buildings. ASHRAE J., nov. 1988: 40-3. [Cópia xerox].

4. HARRISON, J.; FINNEGAN, M.; PICKERING, C.A.C.; AUSTWICK, P.K.C. The sick building syndrome - further prevalence studies and investigation of possible causes. In: Proceedings of the 4th International Conference of Indoor Air Quality and Climate, Berlim, 1987. v. 2 p. $487-91$.

5. HEDGE, A.; STERLING, E.; STERLING, T. Building illness indices based on questionnaire responses. In: Proceedings IAQ-86 Managing Indoor Air for Health and Energy Conservation, Los Angeles, 1986.

6. NATIONAL INSTITUTE FOR OCCUPATIONAL SAFETY AND HEALTH (NIOSH). Guidance for indoor air quality investigations. Cincinnati, Ohio, 1987.

7. SKOV, P.; VALBJORN, O.; PEDERSEN, B.V. Influence of personal characteristics, job-related factors and psychosocial factors on the sick building syndrome. Scan. J. Wk Environ. Hlih, 15: 286-95, 1989.

8. STERLING, E. \& STERLING, T. The impact of different ventilation levels and fluorescent lighting types on building illness: an experimental study. Canad.J. publ. $H h h, 74: 385-92,1983$.

9. STERLING, T. ; COLLET, C. ; RUMEL, D. Epidemiologia dos edifícios doentes. Rev. Saúde públ., S. Paulo, 25:56-63, 1991.

10. WORLD HEALTH ORGANIZATION. Indoor air pollutants: exposure and health effects assessment. Copenhagen, 1982. (Euro Reports and Studies 78).

Recebido para publicação em 1.7.1992
Reapresentado em 17.9.1992
Aprovado para publicação em 28.9.1992 